\title{
Double Swing Quiescent-Current: An Experimental Detection Method of Ferroelectric-like Performance in Very Leaky Dielectric Films
}

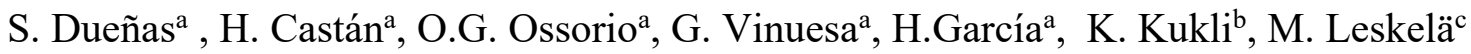 \\ ${ }^{a}$ Department of Electronics, University of Valladolid, Valladolid 47011, SPAIN \\ ${ }^{\mathrm{b}}$ Institute of Physics, University of Tartu, Tartu 50411, Estonia \\ ${ }^{\mathrm{c}}$ Department of Chemistry, University of Helsinki, Helsinki, Finland
}

\begin{abstract}
A new method to characterize the properties of leaky ferroelectric films is proposed. Double swing quiescent-current technique allows to obtain coercive field and remanent polarization in those cases in which high leakage, trap generation current and displacement current mask the ferroelectric terms themselves. Double swing quiescent-current technique allows to detect ferroelectric behavior even in highly conductive materials and obtain reliable values of the remanent polarization and coercive fields which are not affected by the parasitic effects of leakage and displacement currents. In this work, we demonstrate this technique for the case of amorphous $\mathrm{SiO}_{2}-\mathrm{Nb}_{2} \mathrm{O}_{5}$ nanolaminates and mixture films fabricated by atomic layer deposition.
\end{abstract}

\section{Introduction}

Ferroelectric materials are very attractive for many technological applications $[1,2]$. In the scenario of conventional CMOS technology, they are very suitable to fabricate nonvolatile memories (FeRAM) [3,4] or as the gate stack of a transistor to realize a FeFET [5]. Conventional ferroelectric materials, like lead zirconium titanate (PZT) or strontium bismuth tantalite (SBT), exhibit very high permittivity and low coercive field and very thick films must be grown to obtain wide enough memory windows. Because of that, new ferroelectric materials must be investigated. Applications compatible with CMOS technology require very thin layers of dielectrics. Moreover, it is difficult to obtain new insulating materials with low conductivity in the early stages of their technological development. Therefore, ferroelectric properties can be masked by high leakage currents. This can result in the abandonment of these materials before the growing conditions are optimized. In addition, the conventional measurements of ferroelectric properties use techniques in which the electric field is varied with relatively high frequency signals that result in displacement currents that represent an additional term which is added to leakage currents and further conceals ferroelectric behavior. Traditional Sawyer-Tower techniques do not allow the detection of ferroelectric-like behavior when leakage and/or displacement currents are excessively important and even experimental values of polarization may be overestimated when affected by charge terms corresponding to the parasitic phenomena mentioned above. In this work we introduce a new method suitable to overcome these inconveniences and detect ferroelectric-like behavior in leaky materials. First, we demonstrate the validity of this method on conventional ferroelectric materials and afterwards we apply it to silicon oxide-niobium oxide mixture films and nanolaminates grown by atomic layer deposition. 


\section{Experimental method}

The most traditional technique to characterize ferroelectric materials is the measurement of the polarization-electric field hysteresis loop. Usually that is obtained with the SawyerTower method [6] in which the charge released during polarization is stored in a sense capacitor. The total current in a metal-ferroelectric-metal device when a voltage $\mathrm{V}$ is applied is given by the expression:

$$
I(V)=I_{\text {leak }}(V)+I_{t r}(V)+\frac{\partial D(V)}{\partial t}
$$

where $I_{\text {leak }}$ is the leakage current, $I_{t r}$ is the current generated by traps inside the capacitor, and $\frac{\partial D(V)}{\partial t}$ is the current due to the electric displacement. The charge on the sense capacitor is obtained by integrating the current:

$$
Q=\int_{0}^{T} I(V) d t
$$

$D$, is given by [5]:

$$
D=\varepsilon_{0}(1+\chi) E+P_{S}=\varepsilon_{0} \varepsilon_{r} E+P_{S}
$$

where $E$ is the applied electric field, $P_{S}$ is the spontaneous polarization existing in the ferroelectric, $\chi$ is the dielectric susceptibility of the material, and $\varepsilon_{\mathrm{r}}=1+\chi$ is the static dielectric constant, an equation [1] finally results:

$$
I(V)=I_{\text {leak }}(V)+I_{t r}(V)+\varepsilon_{0} \varepsilon_{r} \frac{\partial E}{\partial t}+\varepsilon_{0} \varepsilon_{r} \frac{\partial P_{S}}{\partial t}
$$

In summary, to obtain the spontaneous polarization, $P_{S}$, the three first terms on equation 4 (leakage, trap generated, and displacement current) have to be determined and subtracted from the measured current.

The double swing quiescent-current (DSQC) consists on measuring the current of the sample while varying the applied voltage as follows:

To obtain the positive spontaneous polarization, we apply two identical voltage sweeps from zero to values high enough to polarize the sample. The swing rate is chosen slow enough to consider the measure process as a succession of quasi-stationary states. On the first sweep the current has the four contributions of equation [4]. Afterwards, we repeat the same sweep, but since the material has already been polarized during the first sweep, the component due the spontaneous polarization does not contribute to the measured current. Therefore, the difference between the curves of the first and second sweeps provide the curve due to the polarization current:

$$
I(V)_{\text {sweep } 1}-I(V)_{\text {sweep } 2}=\varepsilon_{0} \varepsilon_{r} \frac{\partial P_{S}}{\partial t}
$$

Once the material is positively polarized, we repeat the same process but applying negative voltage sweeps to negatively polarize the material. 


\section{Results and discussion}

To check the validity of the technique, we have performed previous tests with conventional PZT ferroelectric materials. In Figures 1(a) and (b) we plot the current-voltage and chargevoltage characteristics obtained. We verify that the obtained results match the specifications for these materials.

(a)

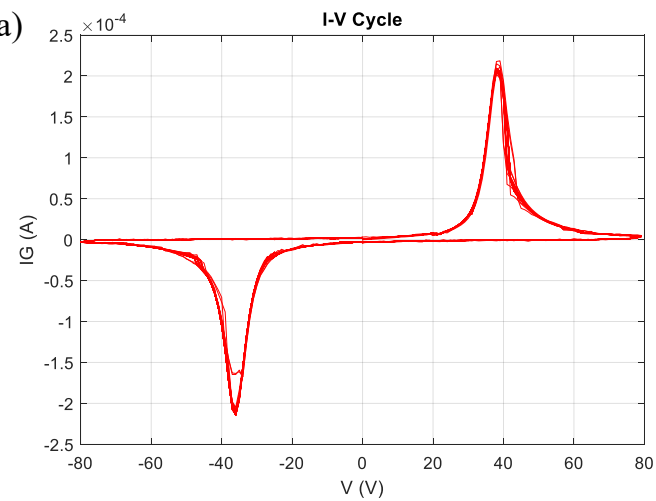

(b)

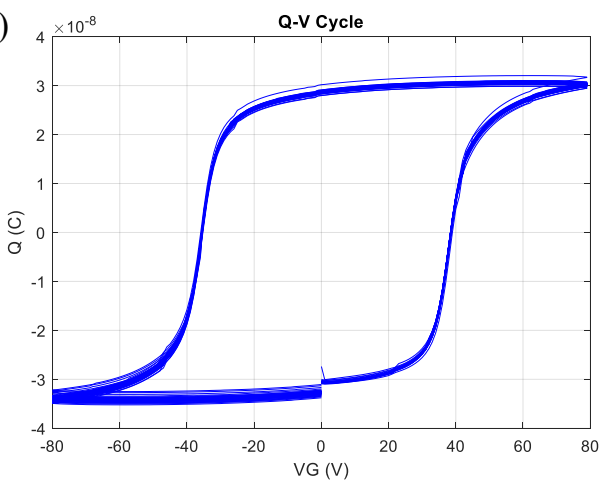

Figure 1. Current-voltage (a) and charge voltage loop obtained for a conventional PZT ferroelectric material.

Afterwards, we have applied the DSQC method to characterize the ferroelectric behavior of amorphous $\mathrm{SiO} 2-\mathrm{Nb}_{2} \mathrm{O}_{5}$ nanolaminates and mixture films. The films were grown by atomic layer deposition. The films were grown at $300^{\circ} \mathrm{C}$ from $\mathrm{Nb}\left(\mathrm{OC}_{2} \mathrm{H}_{5}\right)_{5}, \mathrm{Si}_{2}\left(\mathrm{NHC}_{2} \mathrm{H}_{5}\right)_{6}$, and $\mathrm{O}_{3}$ to thicknesses ranging from 13 to $130 \mathrm{~nm}$. The niobium to silicon atomic ratio was varied in the range of 0.11-7.20. A detailed description of this sample can be found in reference [7].

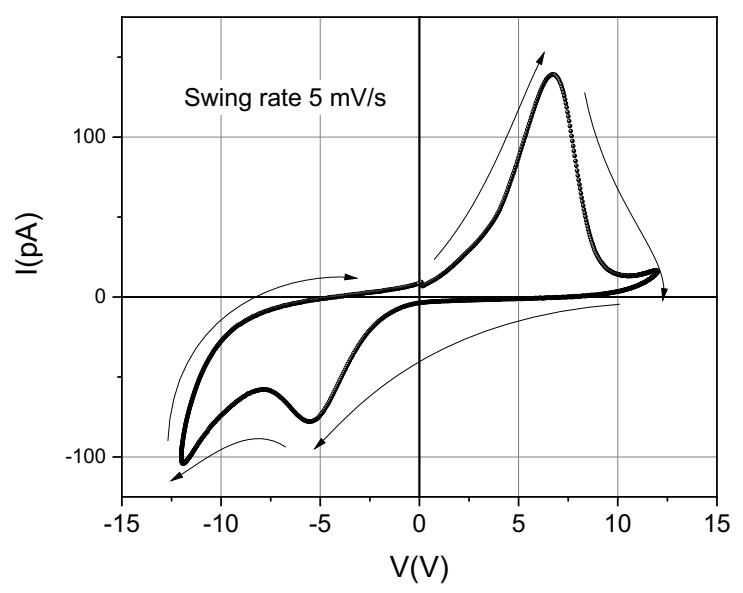

Figure 2. IV loop obtained for a leaky $\mathrm{SiO}_{2} / \mathrm{Nb}_{2} \mathrm{O}_{5}$ capacitor

In Figure 2 we observe a complete voltage current cycle. Starting from $0 \mathrm{~V}$, the voltage increases linearly to a value of 12 volts and then decreases to -12 volts. Finally, the voltage returns to zero. A swing rate of $5 \mathrm{mV} / \mathrm{s}$ has been used to obtain this curve. In this figure you can observe the two peaks corresponding to the positive and negative polarization. It is also noted that the current is not zero when the sample is already polarized due to the existence of leakage and displacement currents. To remove these components two sweeps must be performed in each direction. In Figures 3 and 4 we plot the results of applying 
double sweep for positive and negative voltages, respectively. The difference between the two sweeps provides the ferroelectric polarization current. We note that this current returns to zero once the sample has been polarized.

(a)

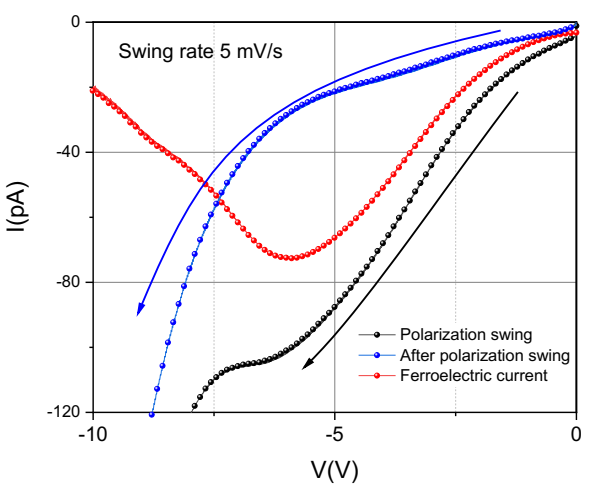

(b)

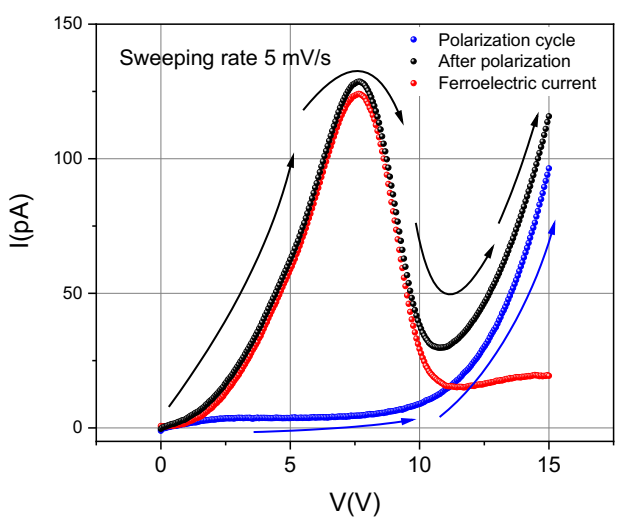

Figure 4. Double swing quiescent current method used to obtain the ferroelectric current at negative (a) and positive (b) electric field for a leaky $\mathrm{SiO}_{2} / \mathrm{Nb}_{2} \mathrm{O}_{5}$ capacitor

\section{Conclusions}

We demonstrate that double swing quiescent-current technique allows to detect ferroelectric behavior even in highly conductive materials and obtain reliable values of the stationary polarization that are not affected by the parasitic effects of leakage and displacement currents. We demonstrate the validity of the method for both conventional and for the case of amorphous $\mathrm{SiO}_{2}-\mathrm{Nb}_{2} \mathrm{O}_{5}$ nanolaminates and mixture films fabricated by atomic layer deposition.

\section{Acknowledgments}

This work was partially funded by the Spanish Ministry of Economy and Competitiveness (TEC2017-84321-C4-2-R), Finnish Centre of Excellence in Atomic Layer Deposition (284623), and Estonian Research Agency (PRG753)

\section{References}

1. L. W. Martin, Y. H. Chu, and R. Ramesh, Mater. Sci. Eng. R. 68, 89-133 (2010).

2. N. Setter, D. Damjanovic, L. Eng, G. Fox, S. Gevorgian, S. Hong, A. Kingon, H. Kohlstedt, N. Y. Park, G. B. Stephenson, I. Stolitchnov, A. K. Taganstev, D. V. Taylor, T. Yamada, and S. Streiffer, J. Appl. Phys. 100, 051606 (2006).

3. O. Auciello, J.F. Scott JF, and R. Ramesh, Phys Today 1998, 51: 22-27 (1998).

4. J.S. Meena, S.M. Sze, U. Chand, and T.-Y. Tseng, Nanoscale Res Lett 9, 526 (2014).

5. H. Ishiwara, in Multifunctional Oxide Heterostructures, E.Y. Tsymbal, E.R.A. Dagotto, C.-B. Eom, and R. Ramesh, Editors, p. 137, Oxford University Press, Oxford (2012).

6. C. B. Sawyer and C. H. Tower, Phys. Rev. 35, 269 (1930).

7. K. Kukli, M. Kemell, M.J. Heikkilä, H. Castán, S. Dueñas, K. Mizohata, M. Ritala, and M. Leskelä, Nanotechnology, accepted manuscript. https://doi.org/10.1088/1361-6528/ab6fd6. 